\title{
Ultrasonic Excitation - Fiber Bragg Grating Sensing Technique for Damage Identification
}

\author{
https://doi.org/10.3991/ijoe.v14i07.8969 \\ Cai Li $\left.{ }^{\bowtie}\right)$ \\ Wuhan Polytechnic, Wuhan, China \\ 1401763814 @qq. com \\ Xiao-Guang Yue \\ Wenzhou Business College, Wenzhou, China
}

\begin{abstract}
Nondestructive testing plays an important role in on-line equipment inspection. Traditional nondestructive testing methods have been widely utilized for this. Ultrasonic excitation-fiber Bragg grating sensing technique is based on well-developed optical fiber grating technology, which has a good prospect of damage detection for mechanical equipments. However the corresponding research is still at the starting stage and further research work is necessary. The main contribution pursued in this investigation is to establish a detection system based on fiber Bragg grating sensing under ultrasonic excitation and predict the damage position through time delay on the plate structure. Differencing from the conventional approaches, a new way of damage detection utilizes fiber Bragg grating sensors and a position algorithm by twodimensional method is derived. Moreover, wavelet transform is adopted in the subsequent signal processing. Finally, a platform for experiment is built to verify the theoretical analysis of the sensing characterization of the fiber Bragg grating sensor in ultrasonic excitation. Experiment results show that the wavelet transform is effective for signal denoising and our localization algorithm is feasible.
\end{abstract}

Keywords-Ultrasonic excitation; Fiber Bragg grating(FBG); Damage detection; Wavelet analysis

\section{$1 \quad$ Introduction}

Large machinery and equipments play a significant complimentary role in many fields, such as aircraft, petrochemical and navy structures. But due to the higher level of the service safety and reliability, the traditional detection methods have certain limitation: On the one hand, damage change has the potential and diversity, the existing detection methods are limited lacking of accurate and effective detection method; On the other hand, the existing each kind of detection methods are susceptible to the influence of various interference factors, which are not very sensitive to corresponding structure caused by material mechanics performance degradation. In the field of aviation, aircraft structural life depends mainly on material life. 
At present, effective means of the damage detection of mechanical equipment as new techniques in recent years are missing. Ultrasonic excitation-fiber Bragg grating sensing technique is based on well-developed optical fiber grating technology, which has been widely used in many engineering fields with the advantages, such as focused power, strong sound press and easily distributed detection network, overcoming the disadvantages of traditional electronic sensors and showing a good application prospect. Some researchers have applied this technique to the damage detection and achieved some degree of success in smart composite structures as well[1]. Duck et al. proposes the theory of two cylindrical model and studied the strain transfer characteristics of the fiber Bragg grating sensor from the base material to the fiber core[2].Hang-Yin Ling et al. proposes a four cylinder strain transfer model for symmetric structures, and studies the strain transfer characteristics from the substrate to the fiber core through the adhesive layer and the coating one[3]. Tsuda et al. verifies fiber Bragg grating (FBG) response sensitivity for ultrasonic wave on the composite materials[4]. Jang, Byeong-Wook uses multiplexed FBG sensor to study positioning algorithm for composite structures[5]. Muthumari reviews of various ultrasonic techniques employed in modern industries[6]. Jang studies real-time impact identification algorithm using fiber Bragg grating sensors for composite structures[7]. Comprehensive analysis of the current research focuses on theoretical research of FBG strain transfer for the mechanism, lacks corresponding experimental verification. In addition, strain transfer theory study of pasted FBG on sheet metal structures also is few. In addition, the research also faces many challenges. Because it is very small, ultrasound strain requires reasonable selection of ultrasonic excitation frequency to ensure the strength of ultrasound strain wave, and to satisfy the sensitivity of FBG detection. The measuring-point arrangement of FBG needs to be studied.

Meanwhile the effective processing methods are expected on the analysis of the ultrasonic signals. Wavelet analysis is an effective signal processing technology, which has been investigated for its transient signal detection capability from structure monitoring[8][9]. The wavelet transform is well suited for extracting transient features out of a nonstationary time series with good signal-to-noise ratio, such as the presented ultrasonic signal.

Therefore, this paper aims to present an FBG sensing system based on ultrasonic excitation for the board plates and the key contributions are as follows. First, the ultrasonic excitation-FBG sensing system is given. It can establish a detection method by combining the advantages of fiber grating sensing with ultrasonic features. Second, the positioning algorithm by an innovative distributed approach is proposed. It can realize two-dimensional position detection of mechanical damage, breaking through the limit of one dimension positionin detection. Third, wavelet transform is adopted, which could ensure the accuracy of the signal recognition.

This paper is structured as follows. Section 2 reviews the basic features of ultrasonic testing and the strain transfer equation of FBG under ultrasonic excitation is given. Section 3 is devoted to damage detection of ultrasonic excitation- FBG sensing system. In Section 4, with the basic experimental verification, our method is proposed and our experiment results show that wavelet analysis for damage detection can effec- 
tively improve the recognition accuracy and the localization method is feasible. Section 5 summarizes the full text.

\section{Ultrasonic excitation theory and sensing strain equation}

\subsection{Excitation theory}

Ultrasonic Lamb wave detection technology is an important nondestructive testing technology for the damage of plate. When the ultrasonic wave encounters an internal defect, where the ultrasonic wave will be reflected, and damage localization can be realized by comparing the waveform changes in different situations. In order to inspect the plate material nondestructively, the principle and structure of an ultrasonic measurement system are described as follows.

In ultrasonic wave active detection, the selection of excitation signal is generally considered from several aspects, such as the incentives, center frequency, and excitation amplitude. Excitation mode of ultrasonic wave mainly has three kinds: ultrasonic transducer, piezoelectric ceramic, and polymer polyvinylidene fluoride (PVDF) excitation. Because it is easier to control the incident angle and the excitation amplitude. So our paper selects ultrasonic probe. Ultrasonic wave is transmitted to the aluminum plate through coupling agent. When the ultrasonic wave frequency is low, there are two kinds of signal modes; While when the center frequency is high, propagation modes of Lamb wave are more complex, and are not conducive to the detect damage signals. The strain caused by ultrasonic wave is small, usually only a few microstrain. Therefore, the appropriate parameters, such as center frequency of the ultrasonic excitation, amplitude of the ultrasonic excitation, are critical in the Lamb wave testing. The ultrasonic excitation and fiber Bragg grating sensing detection based on wavelet analysis is shown in Figure 1.

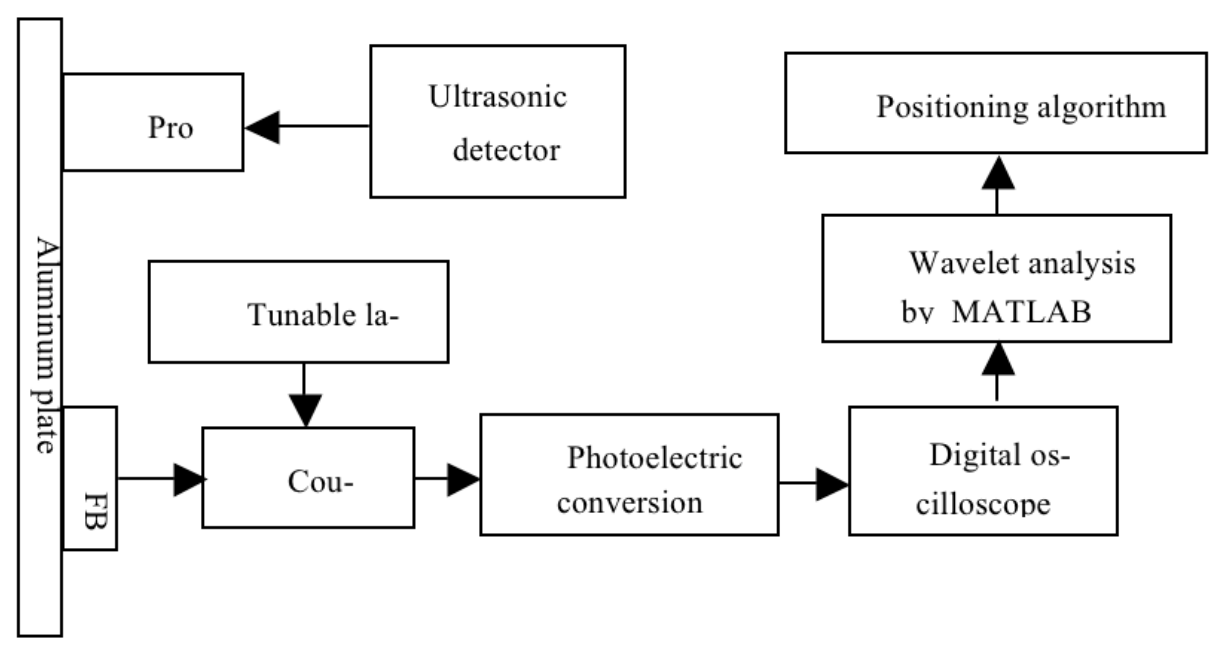

Fig. 1. Ultrasonic detection based on wavelet analysis 
The fiber grating demodulation system, consisting of a tunable laser, a couple and a photoelectric conversion, needs high demodulation speed to realize the measurement of ultrasonic signals. The signals from an FBG demodulation system can be observed with an oscilloscope. They can be analyzed by wavelet transform techniques in software. Eventually our localization algorithm is applied to detect the damage position.

\subsection{Strain Transfer Equation of fiber Bragg grating sensor}

Duck and LeBlanc investigated the mechanical transfer of fibre Bragg grating sensors from a fibre core to a host material by theoretical approaches[2]. Hang-yin Ling investigated strain transfer mechanism of embedded fibre Bragg grating sensors in the composite structure[3], in which the transfer function $H(k)$ of the system is defined as

$$
H(k)=\frac{{\overline{\varepsilon_{z}}}^{f}(k)}{{\overline{\varepsilon_{z}}}^{m}\left(r_{m}, k\right)}=\frac{1}{\left(2 \pi k r_{f} / n\right)^{2}+1}
$$

Where $\mathrm{k}$ is the wavenumber. The symbols $r_{f}$ and $r_{m}$ represent the outer raius of the fibre core and host material respectively from the centre of the fibre core. $\bar{\varepsilon}_{z}^{f}(z)$ $\bar{\varepsilon}_{z}(z)$ are respectively the axial strains of FBG fiber core and base. $\mathrm{n}$ is the strain transfer parameter, which is determined by the interaction of elastic modulus, shear modulus and outer radius of each layer. Equation (1) is derived by the steps addressed in Duck's model and in Hang-yin Ling's model.

In this study, on the basis of the equation 1, we can make the following assumptions on ultrasonic irradiation-fiber Bragg grating sensing detection for the convenience of analysis: first the test material is linear elastic material, which has no plastic deformation, second there is no relative slipping between FBG and the pasted layer; third the deformations of the fiber core are transmitted by shear stress of the substrate through the coating layer and rubber. After a stress analysis on cylindrical model structure of FBG sensor, it ends up with the strain transfer equation of pasted FBG under ultrasonic excitation:

$$
H(f)=\frac{{\overline{\varepsilon_{z}}}^{f}(f)}{{\overline{\varepsilon_{z}}}^{m}\left(r_{m}, f\right)}=\frac{\alpha}{\left(2 \pi f r_{f} / v_{m} n\right)^{2}+1}
$$

Where $\mathrm{f}$ is the ultrasonic frequency, $\alpha$ is the impact factor for pasted FBG, vm is ultrasonic transmission speed. Other parameters are same as above definitions in equation 1. The strain transfer characteristics of the pasted FBG will be affected by 
the impact factor $\alpha$, which is usually less than 1 compared with the embedded FBG. In order to simplify the calculation, we can assume it as a constant.

From Equation (2), we can draw some conclusions: first, if the ultrasonic frequency is high, strain amplitude of FBG is smaller, namely strain perception ability of fiber Bragg grating becomes weaker, and measurement sensitivity becomes lower; Second, the sound pressure on the axis is reduced with the increase of the distance and the optical fiber grating has an axial strain sensitivity: Third, the ultrasonic energy is focused on the acoustic axis. These conclusions from theoretical analysis can be verified in our subsequent experiments in section 4.

\section{Damage Detection of ultrasonic Excitation- FBG Sensing}

Based on the above principles, the monitoring system of the ultrasonic detector is shown in Figure 2. Ultrasonic signals from ultrasonic probe about distance $100 \mathrm{~mm}$ from the central FBG, are transmitted to the plate structure through the coupling agent. FBGs pasted on the board structure serves to detect the ultrasonic strain. The center wavelengths of the FBGs are $1303 \mathrm{~nm}$ and the center frequency of ultrasonic probe is $1 \mathrm{MHZ}$ with a 30 wedge angle. The demodulation system is to demodulate the FBG reflection wavelength, which is displayed by a digital oscilloscope after a10dB photoelectric conversion .Types of tunable laser source is Santec-TSL-510, photoelectric detector is Thorlabs-PDA-10CS, and digital oscilloscope is RIGOL- DS1102E respectively.

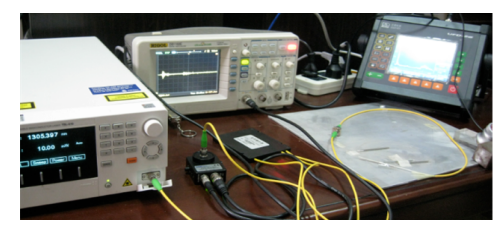

Fig. 2. Ultrasonic detection test

When ultrasonic acoustic axis and optical fiber grating lies on a line, waveform reaches its peak because the optical fiber grating has an axial strain sensitivity and the ultrasonic energy is focused on the acoustic axis. While in the far field, the sound pressure on the axis decreases with the increase of the distance. This phenomenon can be seen from Figure 3. In order to get a better signal ,ultrasonic irradiation angle should be less than 30 degrees. In Figure 3,'1' presents excitation wave,'2' presents reflected wave by plate boundaries. 


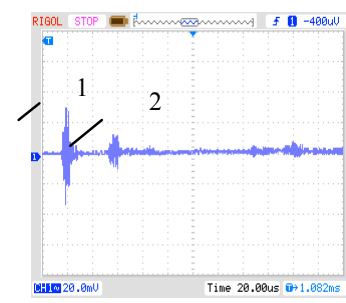

(a)

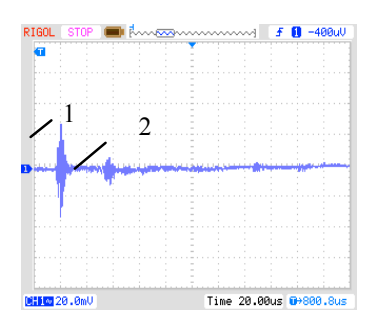

(b)

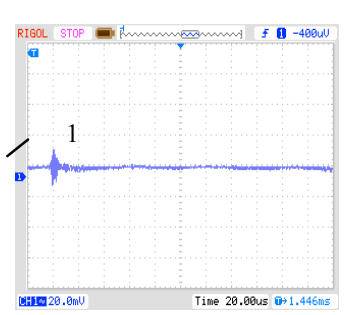

(c)

a- in the 0 degrees ; b- in the 15 degrees ;c- in the 30 degrees 1-Excitation wave; 2-Border echo

Fig. 3. The response waveforms of FBG in the different excitation direction

With the change of incident Angle and frequency, it will produce different signal in different mode at every point. When the ultrasonic waves transmis to the board interface in the incentive area, waveform transformation will happen. After transmission for a period of time in the plate, it will produce the "wave packet" due to the superposition. The curve graph of FBG in different excitation directions or excitation frequencies are respectively shown in figure 4 and figure 5. Take the signal peak as the ordinate axis, it can be obviously seen from Figure 4, the strain peak of FBG decreases significantly with the angle. It can be seen from Figure 5 that under the current inclined block, the $1 \mathrm{MHz}$ frequency is more preferable in ultrasonic damage detection. This is mainly decided by ultrasonic dispersion property.

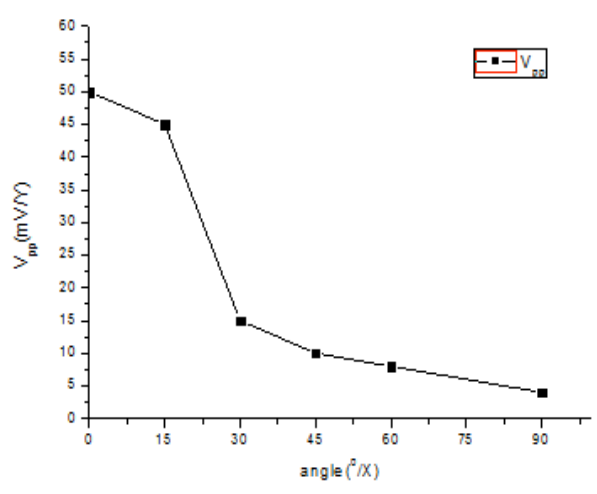

Fig. 4. The curve graph of FBG in different excitation directions

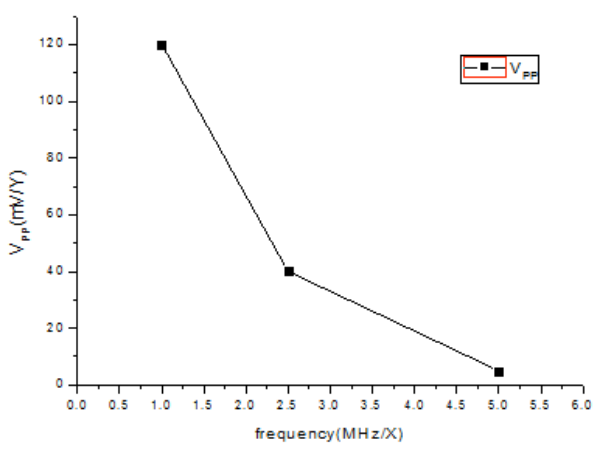

Fig. 5. The curve graph of FBG in different excitation frequencies 


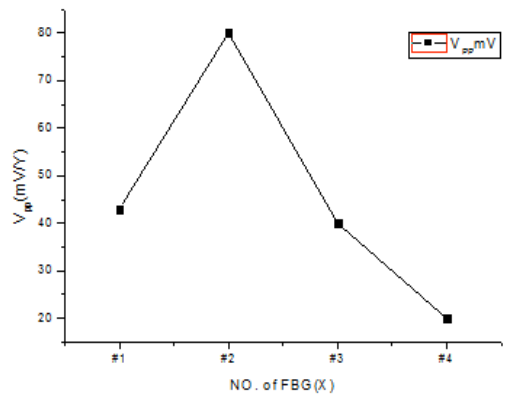

(a)

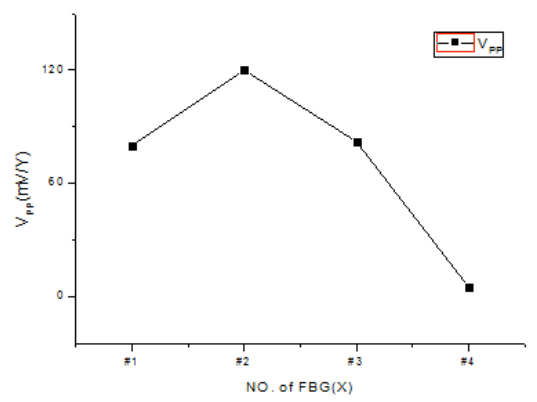

(b)

Fig. 6. The curve graph of FBGs in different detecting position a- Unwashed optical fiber interface b- Alcohol washed optical fiber interface

In Figure 6,corresponding change trends of NO. FBG sensors are consistent, but the amplitude difference is big, such as NO.2 FBG, signal amplitude washed is much bigger than signal amplitude unwashed, nearly 1.5 times. This is mainly due to the influence of light transmission in the optical fiber because of the dust .So it is necessary to clean fiber interface before its use in the common practice. Figure 7 shows the signal contrast of FBG on the healthy and damaged plates. In Figure 7,'1'presents excitation wave, '2'presents reflected wave by plate boundaries and ' 3 ' presents reflected wave by damage on the plate. Their difference is the reflected wave by damage between figure $7 \mathrm{a}$ and $7 \mathrm{~b}$. Then we can find out the reflected wave by damage by comparing the signals on the healthy and damaged plates, then determine the damage position according to the product of the Excitation frequency and the plate thickness in the corresponding mode.

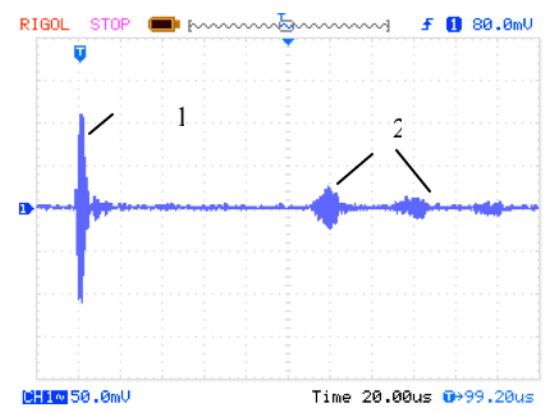

(a)

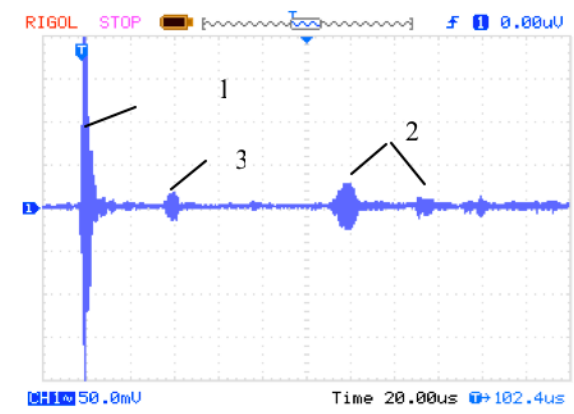

(b)

a- on the healthy plate ; b- on the damaged plate 1-excitation wave; 2-border echo; 3-the reflected wave by damage

Fig. 7. The signal contrast of FBG on the plate 
The above experimental results suggest that this ultrasonic detection allows multiple distributed FBG sensors. However, it forbids such activities as more than the incentive angle 30 degrees of ultrasonic probe. According to the above experimental results, FBG layout and plate dimension is designed as shown in Figure 8. There are four FBGs: \#4FBG is pasted along the acoustic axis with distance $200 \mathrm{~mm}$ from ultrasonic probe, and the \#3FBG are arranged in a vertical line of the acoustic axis, spacing of $50 \mathrm{~mm}$ with the \#4FBG . The distance between ultrasonic probe and the \#4FBG is long and the distance between the \# 3FBG and the 4\# FBG is short. Thus it can ensure that the incentive angle is less than 30 degrees. Figure $8 \mathrm{~b}$ is one of the selected triangle in localization algorithm through the time delay of ultrasonic waves by a simple ellipse algorithm. According to the definition of an ellipse with the excitation probe and the FBG sensor as two focuses, damage point lies on the elliptical circumference. Therefore, we need three distance parameters to determine the elliptic equation: The first one is the distance $d_{i}$ from the excitation source to every sensor, the second one is the distance $r_{i l}$ from the excitation source to damaged position, and the third one is the distance $r_{i 2}$ from the damaged position to the sensor. According to the definition of an ellipse, the distance sum of $r_{i 1}$ pluging $r_{i 2}$ is $l_{i}$. The elliptic equation can be set up by assuming that excitation point is the coordinate origin.

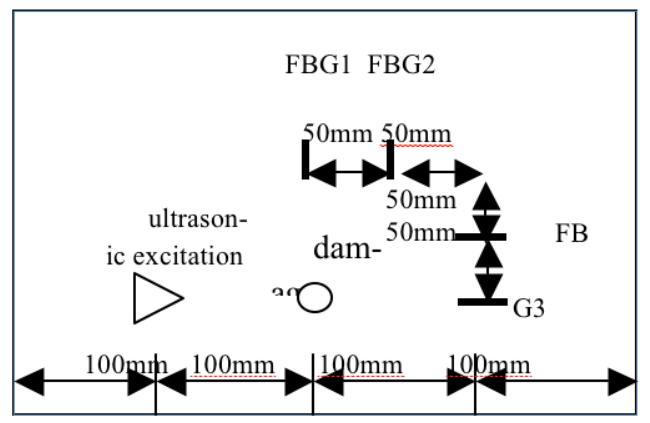

(a)

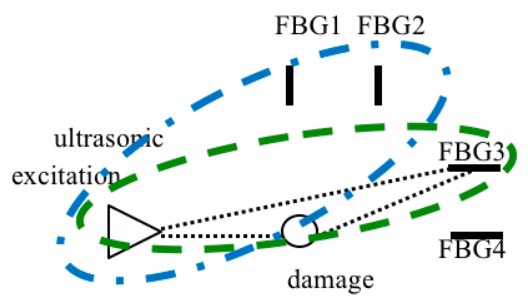

(b)

a-FBG layout and plate dimension b- One of the selected triangle

Fig. 8. FBG layout and plate dimension

An incentive is applied by ultrasonic probe in the position " $\Delta$ ", the waveforms of the FBGs on the health plate are shown in Figure 9. While the waveforms on the damage plate are shown in figure 10. As can be seen from the diagrams in Figure 9 and 10, the signal amplitude of 4 \# FBG reaches its maximum on the shaft of the ultrasonic. The signals of $1 \#$ and $2 \#$ FBG are so small that we can not detect the useful information we need. 
Paper-Ultrasonic Excitation - Fiber Bragg Grating Sensing Technique for Damage Identification

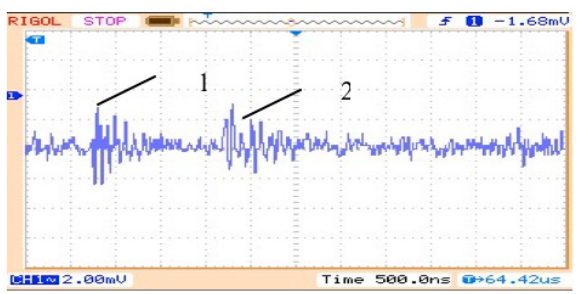

(a)

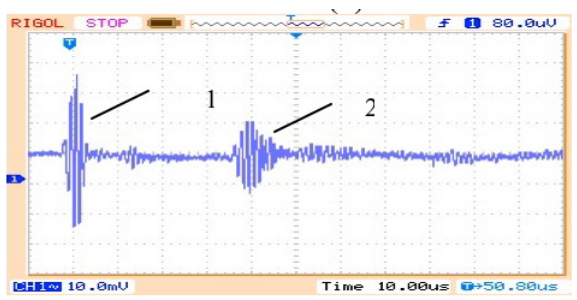

(c)

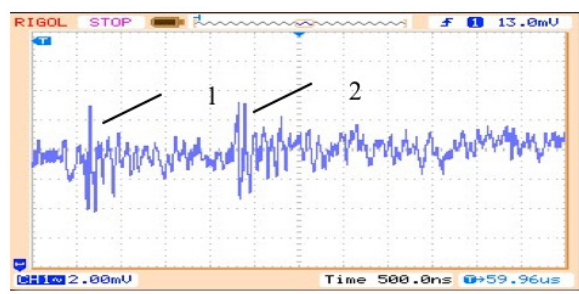

(b)

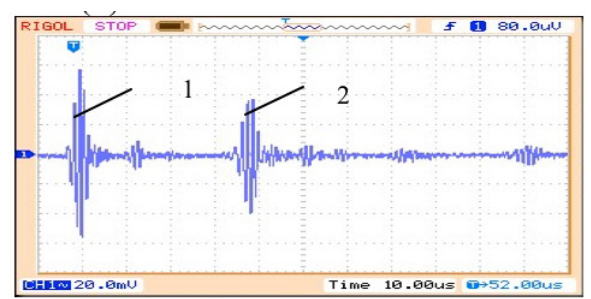

(d)

a-FBG1; b-FBG2; c-FBG3; d-FBG4; 1-excitation wave; 2-border echo

Fig. 9. Signals of sensors on the health plate

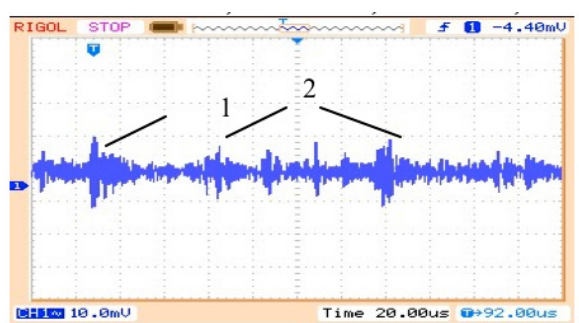

(a)

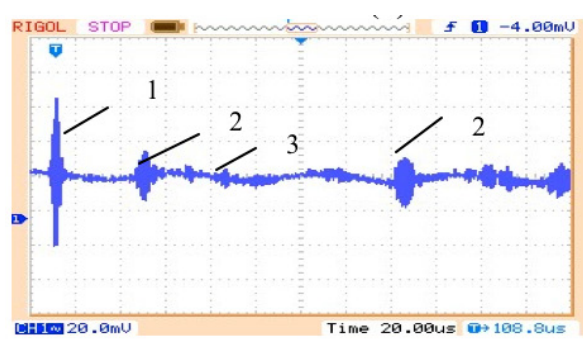

(c)

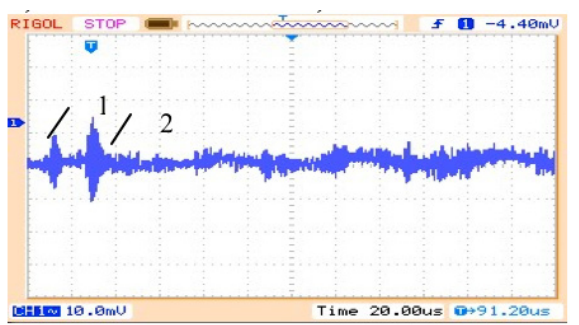

(b)

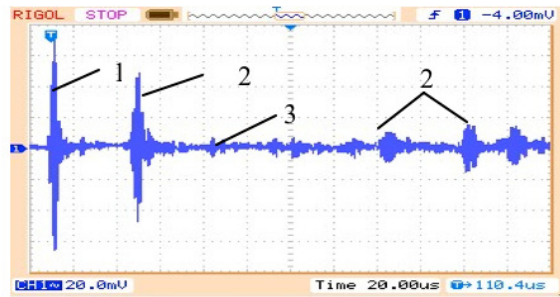

(d)

a-FBG1; b-FBG2; c-FBG3; d-FBG4;

1-excitation wave; 2-border echo; 3 -the reflected wave by damage

Fig. 10. Signals of sensors on the damage plate 


\section{Damage detection based on the wavelet analysis}

Waveform of the FBG on the damage plate has no obvious change compared to waveform of the FBG on the health plate. So, the wavelet analysis tool is needed for signal extraction .First, we begin with wavelet decomposition of the signals containing noise. The wavelet function can be selected as orthogonal wavelets, such as Daubechies $(\mathrm{dbN})$,Symlets (symN), and coiflets (coifN). According to the actual need, a specific choice can be decided. For the ultrasonic signal, it is better to select the compactly supported wavelet bases. To ensure the accuracy on the basis, we select Daubechies (db3) as wavelet basis function, with 5 layers of decomposition in our experiment.

$$
s=a_{5}+d_{5}+d_{4}+d_{3}+d_{2}+d_{1}
$$

In the Equation 3, ai represents decomposition approximation, and di represents detailed part of decomposition, $i=1,2,3,4,5$.The noise is usually included in $d_{1}-d_{5}$. We can process the wavelet coefficients by using threshold, and reconstruct the signal to achieve denoising, as shown in Figure 11. Figure 11a is the signal of $3 \#$ FBG on the damage plate and Figure $11 \mathrm{~b}$ is the signal of 4 \# FBG on the damage plate. Through the decomposition of the signals in Figure 11a, only 3, 4, 5 layer signals can keep the original signal envelope. In the low frequency part, no signal meets the requirements . In Figure 11b, only 2, 3, 4 layer signals can keep the original signal envelope .

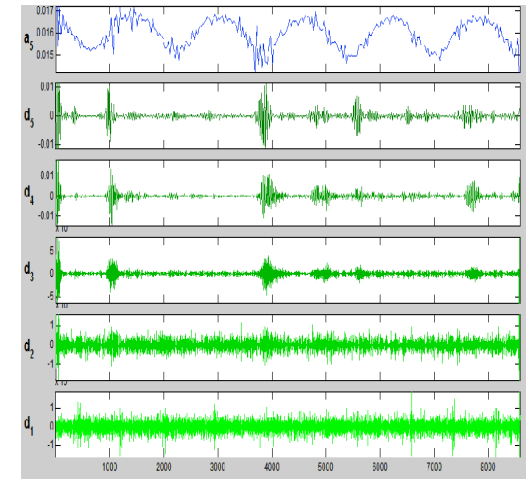

(a)

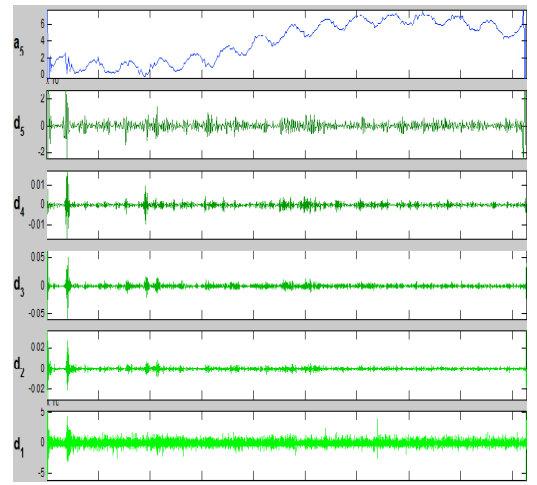

(b)

Fig. 11.Waveform of 3\# and $4 \#$ FBG on the damage plate

a- Signal decomposition of 3\# FBG; b- Signal decomposition of 4\# FBG

Figure 12 is signal comparison of $3 \#$ FBG on the healthy and damaged plates . Daubechies (db3) is selected as wavelet basis function with 5 layers of decomposition. In Figure 12, the first wave packet is electrical signal, which is due to the excitation of the probe producing electromagnetic wave. Electromagnetic wave signal can 
be received at the receiving end in different degrees. The second wave packet is the first reflected wave by plate boundary .In Figure 12b,between the border reflected waves and damage reflected wave ,there is a wave packet in the other mode, which is very small, compared with the excitation signal wave packet. If the signal encounters a defect, the echo signal is also very small. Therefore, wavelet transform is used for denoising processing in order to extract from the acquisition signals containing noises by choosing suitable parameters.

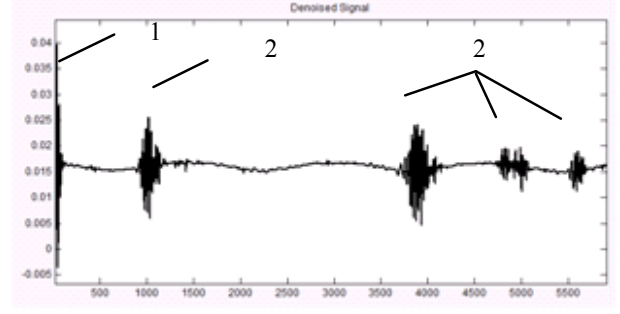

(a)

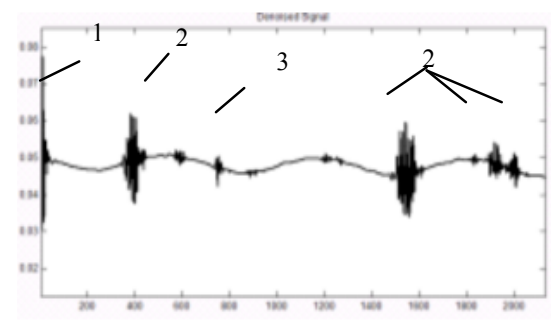

(b)

a- Denoised signal in the healthy plate; $b$ - Denoised signal in the damaged plate 1-Excitation wave; 2-Border echo; 3-The reflected wave by damage

Fig. 12.Signal comparison of $3 \#$ FBG in the healthy and the damaged plates

As illustrated in Figure 9 to 12, the propagation time is 40us when the peak time of the return wave minus the peak time of the excitation signal. Taking 3\# FBG for object, whose sensing signals are collected, we analyze the propagation velocity and delay time of the ultrasonic transmission. In order to establish the elliptic equation,we need to know the distance $d_{i}$ and $l_{i}$, which can be calculated according to the time parameters of the output waveform. Typical healthy waveform obtained by experiment is as shown Figure 9c, and damaged waveform is as shown in figure 10c when the hole diameter of damage is $6 \mathrm{~mm}$. According to comparison of the waveform parameters shown in Figure 10, the elliptic equation is established. Then the second elliptic equation is established by another sensor using the same method. Then we get the intersection of the ellipses, which is the damage position. Assuming that the actual the coordinate position of damage is $(6 \mathrm{~mm}, 24 \mathrm{~mm})$, the coordinate obtained by ellipse method is about $(29 \mathrm{~mm}, 25 \mathrm{~mm})$. The main reason for the difference is due to the ultrasonic mode conversion. The relative deviation is $6 \%$ in terms of the plate size $400 \mathrm{~mm}$. The estimation method of delay time for damage analysis with simple principle is feasible, which realizes two-dimensional position detection of mechanical damage.

\section{Conclusion}

This work focuses on the ultrasonic excitation -FBG sensing technology, which is combined the ultrasonic irradiation penetrability and strong direction with the distributed fiber grating sensing technique. Distributed FBG sensors have been proven to be 
a powerful tool for measurements. In this paper, taking the board structure as the object a detection system of ultrasonic excitation -FBG sensing technology has been given. First, the strain transfer characteristics under ultrasonic excitation are analyzed. Second, the damage location algorithm based on two-dimensional method is studied and the wavelet transform tool is applied. Third, the experimental equipments based on ultrasonic excitation-fiber Bragg grating detection are built to verify the theoretical analysis of the FBG sensor and the phenomena show that wavelet transform is effective by choosing suitable parameters, which promotes accurate extraction from the acquisition signals containing noises, and the estimation method of the location algorithm based on elliptic technology is feasible. This localization algorithm can realize twodimensional position detection of mechanical damage, breaking through the limit of one dimension positionin detection. It has the reference significance to realize mechanical equipment damage location.

\section{Acknowledgment}

This work is supported the following fund:

2017 The project of Hubei Province Natural Science Foundation for Youths (No.2017CFC890).

2017 the Science Research Project of Hubei Province Education Department (No.B2017298).

\section{$7 \quad$ Reference}

[1] Fair Bettayeb, Tarek Rachedi,Hamid Benbartaoui: An improved automated ultrasonic NDE system by wavelet and neuron networks. Ultrasonics , no.42, 853-858,(2004).

[2] Duck Q LeBlanc M: Arbitrary strain transfer from a host to an embedded fibre-optic sensor. Smar-Mater. Struct, vol.9, no.4, 492-497, (2000). https://doi.org/10.1088/0964$1726 / 9 / 4 / 312$

[3] Hang-yin Ling, Kin-tak Lau, Li Cheng and Kwok-wing Chow:Embedded fibre Bragg grating sensors for non-uniform strain sensing in composite structures". Meas. Sci. Technol. vol.16 ,pp. 2415-2424, 2005. https://doi.org/10.1088/0957-0233/16/12/003

[4] Hiroshi Tsuda, Nobuyuki Toyama, KeiUrabe and JunjiTakatsubo: Impact damage detection in CFRP using fiber Bragg gratings. Smart Mater. Struct. vol.13,719-724, (2004). https://doi.org/10.1088/0964-1726/13/4/009

[5] Byeong Wook Jang,Yeon Gwan Lee, Jin Hyuk Kim: Real-time impact identification algorithm for composite structures using fiber Bragg grating sensors. Structural Control and Health Monitoring, vol.19,no.7, 580-591, (2012). https://doi.org/10.1002/stc.1492

[6] S. Muthumari, Anurag Singh: Review of various ultrasonic techniques employed in modern industries: International Journal of Engineering Science and Technology, vol.3, no.4, 3078-3085, (2011).

[7] Jang, Byeong-Wook; Lee, Yeon-Gwan; Kim, Jin-Hyuk: Real-time impact identification algorithm for composite structures using fiber Bragg grating sensors. Source: Structural Control and Health Monitoring, 19(7), 580-591,(2012). https://doi.org/10.1002/stc.1492 
[8] Bao Pengyu, Yuan Mei, Dong Shaopeng, Song Hao, Xueb Jingfeng: Fiber Bragg grating sensor fatigue crack real-time monitoring based on spectrum cross-correlation analysis. Source: Journal of Sound and Vibration, 332(1), 43-57, ( 2013).

[9] Panopoulou A., Roulias D., Loutas T.H., Kostopoulos V.:Health monitoring of aerospace structures using fibre Bragg gratings combined with advanced signal processing and pattern recognition techniques. Source: Strain, 48(3), 267-277, (2012). https://doi.org/10.1111/j.1475-1305.2011.00820.x

\section{Authors}

Cai Li (1980-), come from Wuhan, Hubei province. Ph.D.degree, the main research direction for equipment detection and diagnosis. (e-mail: 1401763814@qq.com, affiliation: School of Mechanical and Electronic Engineering, Wuhan Polytechnic, Wuhan, 430070, China).

Xiao-Guang Yue (1986-), come from Wuhan, PhD and Associate Professor, the main research direction for Intelligent Information Processing. (email: xgyue@foxmail.com, affiliation: Wenzhou Business College, Wenzhou, 325035, China).

Article submitted 16 October 2017. Resubmitted 29 November 2017. Final acceptance 15 January 2018. Final version published as submitted by the authors. 